\title{
Vibrotactile Feedback in the context of Mu-rhythm Based BCI
}

\author{
Febo Cincotti, Laura Kauhanen, Fabio Aloise, Tapio Palomäki, Nicholas Caporusso, Pasi Jylänki, \\ Donatella Mattia, Fabio Babiloni, Gerolf Vanacker, Marnix Nuttin, Maria Grazia Marciani, \\ and José del R. Millán.
}

\begin{abstract}
Brain-Computer Interfaces (BCIs) need an uninterrupted flow of feedback to the user, which is usually delivered through the visual channel. Our aim is to explore the benefits of vibrotactile feedback during users' training and control of EEG-based BCI applications.

An experimental setup for delivery of vibrotactile feedback, including specific hardware and software arrangements, was specified. We compared vibrotactile and visual feedback, addressing the performance in presence of a complex visual task on the same (visual) or different (tactile) sensory channel.

Results indicated that the vibrotactile channel can function as a valuable feedback modality with reliability comparable to the classical visual feedback. Advantages of using a vibrotactile feedback emerged when the visual channel was highly loaded by a complex task.
\end{abstract}

\section{INTRODUCTION}

$\mathrm{T}$ HE human brain relies on inputs from different senses to form percepts of objects and events, during everyday life. These pieces of information usually complement and confirm each other, thereby enhancing the reliability of percept [1]. Somatosensory feedback is a vital component of motor planning, control and adaptation, and there is a growing effort to include this feedback modality in neural prosthetic systems [2].

Visual presentation of stimuli is the most common feedback modality in neurofeedback paradigms for selfregulation of the brain's electrical activity. Thus, it is comprehensible that current brain-computer communication systems mainly operate with visual stimuli [3]. However, components of the visual system such as vision, visual

Manuscript received April 16, 2007. This work has been partially supported by the European IST Programme FET Project FP6003758.(MAIA). This paper reflects only the authors' views and funding agencies are not liable for any use that may be made of the information contained herein.

F.C., F.A., N.C., F.B., and M.G.M. are with the Laboratorio di Imaging Neuroelettrico e Brain Computer Interface, Fondazione Santa Lucia, IRCCS, Rome, 00179, Italy. (+390651501466; +390651501465; f.cincotti@hsantalucia.it).

F.B. i salso with the DFUF, Università degli Studi di Roma "La Sapienza", Italy

M.G.M. is also with the DN, Università degli Studi di Roma "Tor Vergata", Italy

L.K, T.P. P.J are with the LCE, Helsinki University of Technology, Finland.

M.N. and G.V. are with DME, Katholieke Universiteit Leuven, Belgium

J.M. is with the IDIAP Research Institute, Martigny, Switzerland. and with the Ecole Polytechnique Fédérale de Lausanne (EPFL), Switzerland attention, focusing gaze are physiologically engaged during the dynamic contact between the body and environment. Furthermore, the visual sense may be compromised on some patients who are in need of BCI support. Thus, towards more efficient brain-computer communication, it seems important to also obtain evidence of how the extra-vision somatosensory modality performances during self-regulation of the brain's electrical activity.

Only few studies have tested other feedback modalities for brain-computer interfaces (BCIs). Hinterberger et al [4] and Pham et al. [5] tested auditory feedback, but, to our knowledge, no one has trained subjects with tactile feedback. In addition to freeing visual and auditory attention, tactile stimuli are more natural in a manipulation task than, e.g. visual stimuli. Even though BCI training is strongly dependent on feedback, surprisingly, only two studies have explored how feedback affects the learning process. McFarland et al [6] investigated what happens when feedback is removed from well-trained subjects and Neuper et al [7] compared continuous and discrete feedback.

This study aims to explore the benefits of vibrotactile feedback for user training and accurate control of an EEGbased Brain Computer Interface. To this purpose, we mimic a "real-life" condition wherein subjects were engaged in a complex visual task (which requires focused visual attention) and simultaneously they received the necessary continuous information about the status of the system they are using. BCI trained subjects were thus, exposed either to a visuo-visual or to a visuo-vibrotactile feedback of the outcome of BCI control and overall task, respectively, to assess whether the vibrotactile information may effectively complement the visual channel.

\section{METHODS}

\section{A. Vibrotactile Stimulus Procedure}

C-2 Tactors (Engineering Acoustics, Inc, Winter Park, FL, USA) are magnetic actuators, similar in principle to audio speakers; the current flowing in its coil pushes a central structure named contactor against the skin and back. Different from acoustic transducers, the structure is tuned on a narrow band around $250 \mathrm{~Hz}$, so only signals at these frequencies can be effectively transduced. By driving a tactor with two mixed sine waves, complex waveforms can be obtained. Moreover, a third auxiliary input can be fed at the amplification stage. Even if efficiency issues suggest not 
to deviate from the resonance frequency of $250 \mathrm{~Hz}$, the frequency of stimulation can be selected by the user. The output intensity can be set to four different values (amplification gains). A Peripheral Interface Controller (PIC) included on the control board takes care of serial communication with the PC, and sets in the generation and amplification subsystems the appropriate values of frequency and gain. By using a battery as power supply and a serial port to a Bluetooth (BT) adapter the host PC can send commands over the air to the vibrotactile device. Since both the control board and the BT adapter are battery powered, the users can wear a wireless system during the experiments. The tactors are relatively lightweight and small ( $\sim 4 \mathrm{~cm}$ diameter). Eight tactors were positioned in a circle at even angles on the upper part of the trunk of the subjects. The tactors were placed over a t-shirt, and kept in place by a second elastic t-shirt, which also provided the necessary preload. Vibrotactile stimuli were given at $250 \mathrm{~Hz}$, lasting $500 \mathrm{~ms}$. During a testing session, 256 separate stimuli were delivered to each subject, in four runs separated by short breaks. Each stimulus could be given to one of the eight tactors, and could have an intensity level of one to four. All subjects reported that they could perceive the stimulation, even at the lowest intensity. The average response time (two key presses, direction and amplitude) was $2.35 \pm 0.52 \mathrm{~s}$. Overall errors in detecting the correct position were 3.8\% and errors in detecting the intensity of stimulus were $35.9 \%$. In both conditions, $0.2 \%$ of the responses were not classified. Most of the errors were made with stimuli delivered to the right part of the body, and for intermediate intensities.

\section{B. Subjects and task procedure}
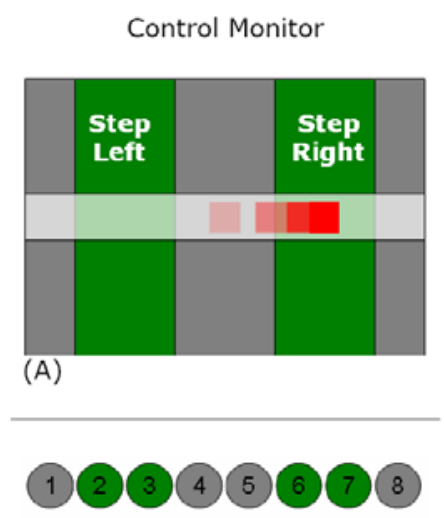

(B)

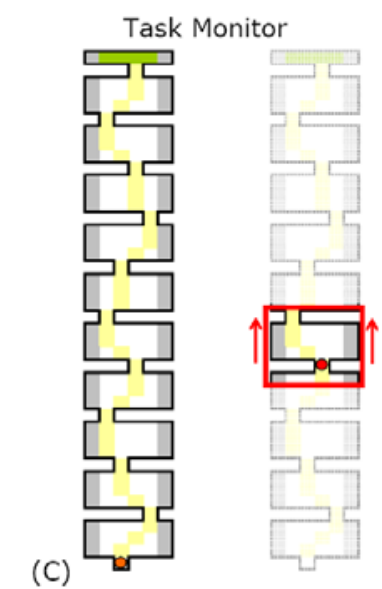

Fig. 1 Panel A: visual feedback of the pseudo-BCI controller; the subject had partial control on the red cursor, whose position was converted at discrete times (2 s) into navigation commands (step left, right or no stepping). Panel B: vibrotactile feedback of the pseudo-BCI controller; each tactor of the stripe encoded the tactile version of the visual cursor. Panel C: scheme of the task; the drawing to the left represents the whole maze, with the ideal path marked in yellow. In the drawing to the right, the scrolling red frame shows the portion of the maze visible at once of the task display.
Subjects were exposed to a joint visual and vibrotactile feedback, to assess whether the vibrotactile information could complement the visual channel for feedback, when the visual channel is intensively used as a carrier of task-related information. This experimental condition mimicked a "reallife" condition where the subject, engaged in a given task (spatial navigation), could simultaneously receive continuous information of his control strategy (feedback). Continuous state of the control signal, rather than a timediscrete classification was fed back to the user. To better focus on the properties of feedback and to reduce intersubject and inter-session variability (due to different level of training and fatigue), the "BCI" control signal was not derived by modulation of subject's brainwaves, but simulated by the movement of a PC mouse, to which a BCIderived noise was added.

Thirteen voluntary subjects, two of which suffered from paraplegia due to lesions to their spinal cord, were involved in the experimentation. The experimental task consisted of moving a placeholder visible on a "Task" monitor, with the goal of stepping through a sequence of 10 "rooms" (Figure 1), following a path constrained by narrow "gates" between adjacent rooms.

\section{Control monitor}

Subject's intention to move the placeholder was mediated by a BCI-like controller. In a first setting, the visual feedback of this controller was visible in a "Control Monitor" (Figure 1.A). The horizontal position of a cursor was partially regulated by the subject, moving a computer mouse. In fact, the cursor movement was affected by noise and delay, so that (inaccurate) motion was as similar as possible to a typical BCI-controlled cursor trajectory. To achieve this goal, the processing chain of the BCI2000 software [9] was setup like in a mu-rhythm-based cursor control task, except for the fact that the amplitude of the spectral "EEG" component of interest was modulated by the mouse position; also, the time-series of cursor drift from an actual EEG-modulation recording was added sample by sample to the cursor control signal.

In a second setting, the feedback of this BCI-like controller was given through a stripe of eight tactors (Figure 1.B), positioned on the shoulders of the subject as shown in Figure 2A. Only one tactor at a time was active, encoding information about the horizontal position of a tactile cursor.

Once every $2 \mathrm{~s}$, the (visual or tactile) cursor's horizontal position was sampled and compared to the limits of the five intervals defined on the screen; and the placeholder moved one step to the right, to the left or stayed in its line, accordingly (see Figure 1.A and B). If not impeded by a transverse "wall", the placeholder moved one step ahead at each time. Since the extreme (left and right) position of the control cursor did not produce a lateral movement of the placeholder, the subject could not simply grossly move the cursor in one direction, but had to attend the visual feedback on the Control Monitor, to make sure he did not under- or 
over-shot cursor's position (which would be too an easy control strategy). This designed produced (i) the need of attentive level, and (ii) a number of mistakes that were comparable to real BCI operation. Subjects practiced for 30 min with the Control Monitor alone (both visual and tactile) to stabilize performance before challenging the task.

\section{Task monitor}

Each room of the navigation space measured $4 \times 4$ steps and access to the following room was allowed only through a narrow "gate". In the task monitor, movement was strongly discretized (one step every 2 seconds), so that the subject could not infer the status of the controller by looking at the placeholder's motion.

To force subjects to keep their visual attention on the Task Monitor, a colored (green or yellow) key appeared at random times once or twice for each "room". Before proceeding to the next "room", the subject had to report the color of the last key. If wrong, the subject had to navigate again the same room, thus making the path to the final goal longer (and more time consuming). Subjects had to perform six runs of the task. The visual or the vibrotactile feedback was provided in alternate runs. Type of feedback of the first run was randomized across subjects.

Control commands and navigation trajectories were recorded, and several indices of performance were computed offline: rate of steps in the ideal path (SIP), rate of steps in an acceptable path (SAP), time to complete the 10 room path, rate of correct answers to the attentional task (key color).

T-test was performed on these indices to compare the effects of visual vs. tactile feedback.

\section{RESULTS}

The rate of steps within the ideal path was comparable in the two conditions ( $80.9 \%$ vs. $83.7 \%, \mathrm{p}>0.05)$. Considering slightly swinging trajectories around to the ideal path as acceptable, visual feedback allowed higher performance $(92.1 \%$ vs. $89.2 \%, p=0.004)$. Nevertheless, the number of

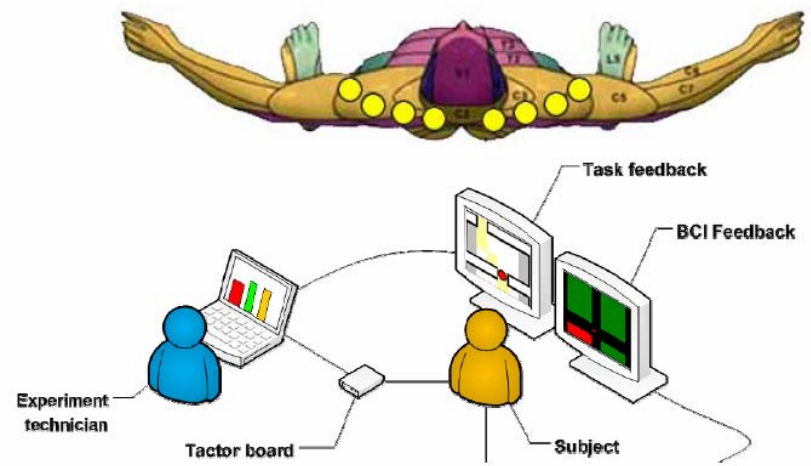

Fig. 2 Panel A):positions of the stripe of tactors on the subject's shoulders. Panel B):experimental setup for visual feedback; the monitors in front of the subjects show the navigation task (Task Monitor, top) and the pseudoBCI feedback (Control Monitor, bottom). keys incorrectly reported is clearly higher during the runs with visual feedback $\left(86.0 \%\right.$ vs. $\left.97.5 \%, \mathrm{p}=10^{-4}\right)$. Given the payload set for wrong answer, this yielded a significantly longer time to destination in the same condition (182 s vs. $131 \mathrm{~s}, \mathrm{p}=2 \times 10^{-4}$ ). Remarkably, two of the subjects reported appearance of blue and red keys (which were never delivered), only during runs with visual feedback.

\section{DISCUSSION}

The tactile feedback modality was used and compared to the visual while subjects were required to perform a visually guided navigation task. We reduced the experimental variables, by setting up a pseudo-BCI control, which retains the typical inaccuracy, delay, and attention requirements of an EEG-based BCI.

If we only consider the ability of subjects to guide the placeholder towards the gates, the accuracy obtained with visual and tactile feedbacks was comparable. A deeper analysis, showed that with tactile feedback subjects tend to stay closer to the ideal path, thus pacing on a more straight line. The most notable difference was in the attentive resources that subjects were able to devote to the task. A significantly higher rate of mistakes was made when visual attention was divided between the Control and Task Monitors.

The subjects reported a good level of comfort in the experimental session lasting about 1 hour. Prolonged test are needed to assess long-term compliance.

The importance of feedback in BCI experimentation is unquestionable, both during the training phase, and at a later stage. Though visual feedback, which exploits the human's richest sensory channel, is most exploited in this field of research, in this experimental series we tested how well we can convey an appropriate flow of information into vibrotactile stimulation. To this purpose we developed a hardware system and a set of software programs that were interfaced to a BCI setup. Information from the BCI system was successfully translated into tactile stimuli, exploiting the features of the sensory channel that physiology are best detectable by users.

In the experiments we conduced, tactile feedback does not interfere with simultaneous visual stimuli and it may improve performance when the subject's attention is highly loaded by a simultaneous visual task.

Although these observation have to be confirmed on a larger scale of experimentation (more subjects), it is conceivable to assume that the vibrotactile channel can be effective in relieving the visual channel whenever a dynamic environment overloads the visual channel. In fact, as in the last experimental setting, the user of a BCI system in a reallife context should be able to attend the continuous incoming information both from the BCI feedback itself and the task-relevant information (i.e. navigation information, unexpected obstacles, directions) which would mostly be mediated by his/her visual sense. This information 
processing requires at this stage, a very high level of attentional effort and decrease of performance is likely to occur if this sensory load is not divided onto different senses. In this regard, future experiments are needed to explore the natural integration between multimodal feedbacks (visual, auditory, and tactile) in oriented tasks executed under BCI control. Vibrotactile feedback could be of practical use in applications of BCI technology. Not only it would allow a user to receive a private feedback message (i.e. not perceivable by people close to him), but it could be packaged into a wearable device and hidden under clothes, thus improving portability of the system.

\section{CONCLUSION}

In conclusion, our findings indicate that the vibrotactile channel can function as valuable feedback modality in a BCI-controlled setting. Its reliability is comparable to the classical visual feedback, and it can improve performance during tasks that need a focused visual attention.

\section{REFERENCES}

[1] B.E. Stein and M.A. Meredith "The Merging of the Senses," -224, 1993.

[2] A.B. Schwartz, X.T. Cui, D.J. Weber and D.W. Moran "Braincontrolled interfaces: movement restoration with neural prosthetics," Neuron JT - Neuron vol. 52, no. 1, pp. 205-220, 2006.

[3] J.R. Wolpaw, N. Birbaumer, D.J. McFarland, G. Pfurtscheller and T.M. Vaughan "Brain-computer interfaces for communication and control," Clin Neurophysiol vol. 113, no. 6, pp. 767-791, 2002.

[4] T. Hinterberger, N. Neumann, M. Pham, A. Kubler, A. Grether, N. Hofmayer, B. Wilhelm, H. Flor and N. Birbaumer "A multimodal brain-based feedback and communication system," Exp Brain Res vol. 154, no. 4, pp. 521-526, 2004.

[5] M. Pham, T. Hinterberger, N. Neumann, A. Kubler, N. Hofmayer, A. Grether, B. Wilhelm, J.J. Vatine and N. Birbaumer "An auditory braincomputer interface based on the self-regulation of slow cortical potentials," Neurorehabil Neural Repair vol. 19, no. 3, pp. 206-218, 2005.

[6] D.J. McFarland, L.M. McCane and J.R. Wolpaw "EEG-based communication and control: short-term role of feedback," IEEE Trans Rehabil Eng vol. 6, no. 1, pp. 7-11, 1998.

[7] C. Neuper, A. Schlögl and G. Pfurtscheller "Enhancement of left-right sensorimotor EEG differences during feedback-regulated motor imagery," J Clin Neurophysiol vol. 16, no. 4, pp. 373-382, 1999.

[8] G. Schalk, D.J. McFarland, T. Hinterberger, N. Birbaumer and J.R. Wolpaw "BCI2000: a general-purpose brain-computer interface (BCI) system," IEEE Trans Biomed Eng vol. 51, no. 6, pp. 1034-1043, 2004 